\title{
Right Inferior Frontal Convolution
}

National Cancer Institute

\section{Source}

National Cancer Institute. Right Inferior Frontal Convolution. NCI Thesaurus. Code C33478.

A gyrus on the outer surface of the right frontal lobe of the cerebrum located between the inferior frontal sulcus and the fissure of Sylvius that is involved in response inhibition. 